\title{
Drawing places, recreating spaces: visual voices from at-risk children
}

\author{
Giuliana Pinto (i) ${ }^{1}$, Francesco Tosi ${ }^{1} \&$ Oriana Incognito (iD ${ }^{1 \times}$
}

Drawing is a highly participatory mode of communication, particularly suited to allowing children to express their knowledge and ideas about various aspects of reality. It is necessary to ascertain whether children are able to master drawing sufficiently to place it at the service of their representational intentions, and whether they possess the pictorial flexibility needed to articulate and differentiate their graphic representations. The presence and development of this important cognitive-symbolic ability are investigated. This exploratory study aimed to investigate, in children living in socioeconomic and cultural disadvantaged conditions, (1) the emergence and development of the pictorial flexibility needed to effectively represent and differentiate the building in which they live from the building in which they wish they lived; and (2) the patterns in the number and quality of pictorial differentiation strategies adopted for representational purposes. Two-hundred 8-to-12-year-old Brazilian children living in a favela were asked to produce two specific thematic drawings, representing their real house vs. their desired house. The children's pictorial representations were coded according to their communicative efficacy (allowing the viewer to distinguish, in each pair of drawings, between the real house and the one desired by the drawer) and according to the number and type of pictorial strategies used to diversify the two types of buildings. The children were had sufficient representational flexibility to effectively perform a pictorial differentiation task, and express their point of view on the environment in which they live, and imagine alternative scenarios, adopting a variety of painting strategies. Drawing, prompted with a contrastive task, has proven to be effective in allowing even disadvantaged children to differentiate their real and desired urban environments. Children's pictorial flexibility manifests itself through a wide range of strategies, varying in number and quality according to the age of the artists. As such, its use can be encouraged by educational interventions aimed at broadening the expressive potential of children, and as a tool for fostering resilience.

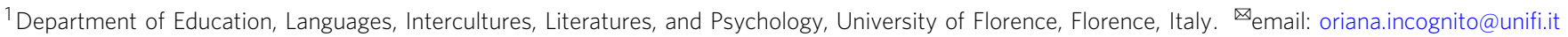




\section{Introduction}

Drawing and resilience. Resilience can be broadly defined as the capacity of a dynamic system to adapt successfully to disturbances that threaten system function, viability, or development (Masten, 2014). Resilient children emerge from stressful environments, take active roles in their lives, and realize their accomplishments: self-efficacy, competency, independent thinking, autonomy, communication skills, problem-solving, and the capacity to manage strong feelings and impulses (Rutter, 1985). Several additional factors are associated with resilience, such as caring and supportive relationships within and outside the family (Chapin, 2015). Resilience can also be supported by providing children with communicative means and symbolic activities that can empower them by encouraging their feelings of participation (Eisner, 1982). Among the symbolic activities supporting resilience, drawing has been proven to occupy a favorable position (Mento et al., 2019).

Psychological research has increasingly focused on drawing as a purposeful and powerful tool for children's meaning-making, recognizing, interpreting, and transforming reality (Ring, 2006); for adults, it offers an alternative way to access children's views and experiences, which can be used with children more easily and cheaply than verbal instruments (Clark, 2005), especially through different populations (Huss and Alhaiga-Taz, 2013; NuttmanShwartz et al., 2010; Pinto and Bombi, 2007). In this vein, numerous interventions aimed at gaining children's perspectives on the difficult circumstances in which they live have made use of thematic drawing. A number of these specifically directed children to draw their living environment, everyday places (both urban, architectural), and natural landscapes. The choice of this pictorial theme is firmly rooted in the tradition of research into children's drawing: developmental psychology has emphasized how a child's emotional attachment to a place is an important part of his or her identity (Winnicott, 1987), which includes memories, feelings, and preferences expressed in relation to physical spaces that have been places of life (Fried, 2000). Architectural structures have a considerable influence on the well-being of individuals, entering into close interaction with their perceptual and cognitive processes, social skills, and the sphere of their emotions (Proshansky and Fabian, 1987). According to Matthews (1985), drawing is the best tool for children to express their ideas about the buildings and architecture around them and for researchers to capture children's perspectives in understanding ideas and practices of "home." In the study performed by Aysan and Oliver (2001), the house that was drawn proved to be a significant indicator of the ways in which spaces are respected and utilized, the degree of privacy or security that the dwelling affords, the number of people that occupy it, their domestic relationship, and responsibilities to each other of its inhabitants. Visual patterns of the houses drawn are effective clues to children's image of themselves and their relationship with family and friends, according to Cherney et al. (2007). Izadkhah and Gibbs (2015) asked 202 preschool children, aged 5-6 years, from four districts of Tehran (Iran) to draw pictures of lands affected by earthquakes. The features that were observed in the children's drawings included using color to indicate anxiety and fear; using parts of the body when taking shelter from earthquakes; providing assistance as well as seeking it during times of disasters; and appropriate (safe) and inappropriate (unsafe) places to shelter. In a study involving children who were caught in Hurricane Katrina in the United States, Kim (2005) observed how the child's perception of the disaster was expressed through a visual representation of the storm, disease, corruption in the environment, and people's feelings of loss, offering her representation of the disaster as a combination of manmade, natural, and individual disasters. In a case study, focusing on one thriving migrant Thai adolescent during a filmed "day in his life" (Cameron et al., 2014), the participant, 14-year-old "Pond", drew spontaneously for more than an hour and proudly shared his drawings with the father as well as with the researchers. His graphic endeavors, representing domestic and urban landscapes, included traditional Thai representations and pop-cultural cartoon sketches, bridging the worlds he navigated as he adapted to his new domicile. In their study, Fargas-Malet and Dillenburger (2014) explored, through a draw-and-tell technique, children's perceptions of their own lives and their predecessors' lives in Northern Ireland, where regular riots and paramilitary activity continue the division and conflict. While multiple positive elements of peace and hope were depicted by most children, especially in the pictures portraying the present, negative elements and violent references mostly appeared in the pictures representing the past.

Taken together, these studies suggest that drawing their life environment offers a way for children to make sense of their experiences, to express grief and loss, and to become active participants in their own process of healing. However, the range and variety of experiences and most of the communications on this topic are narrative and descriptive in nature rather than research-oriented. For this reason, it is difficult to get a thorough picture of how drawing the setting in which they carry out their daily life is used with children living in difficult circumstances. Therefore, more reliable research and planning is needed, as Orr (2007) concludes in his review.

Drawing to communicate: pictorial flexibility. Most of the interventions used drawings of the buildings produced spontaneously or in response to very general requests, sometimes in a clinical setting by children directly involved in adverse conditions. This method of collecting pictorial material allows a great deal of arbitrariness in interpretation by the recipient (whether psychological or educational professional) and leaves open an important methodological question: Is the viewer in front of a specific drawing of the required theme, inspired and modeled on the crisis, or is it a generic representation, the one that a child would have drawn anyway, even under normal conditions? To overcome this obstacle, the use of contrary pictorial tasks has proven to be particularly valid, that is, in which the children are asked to perform two different representations of the same pictorial object, each illustrating specific characteristics of the depicted theme. This collection method has proven fruitful in soliciting diversified representations of various themes, such as the child's interpersonal relationships (Laghi et al., 2013), self-image (Bombi and Cannoni, 2003), and school life (Pinto et al., 2011). This method requires the drawers to use their pictorial flexibility, but it is not an easy task: according to Thomas and Silk (1990), graphic development can be assimilated to the discovery of rules and regularities that underlie the construction of informative and recognizable representations, shared by the "visual" community the child belongs to. Whenever a child is asked to draw, there will be a tendency to rely on traditional schemata for the representation of ordinary objects (human figures, animals, buildings) shown from a typical view, one that best displays their important structural or invariant features. These typical, "canonical" representations allow children to reach a great communicative efficacy through limited executive effort, because few signs (and simple ones) are often required to make the drawn objects recognizable by others. As children develop, they learn to adjust to changing demands and priorities to consider something from a fresh or 
different perspective (Barrett and Light, 1976). To depart from these traditional, stereotyped representations and connote the pictorial object in a more personal way, children must have what is referred to as "cognitive flexibility". As Karmiloff-Smith (1990) indicates, this will come about through a spontaneous interfacing with the child's own internal descriptions (compiled procedures), initially through intra- and, eventually, inter-representational flexibility. In her representational redescription model, "representational redescription" is defined as the mental processes through which a child produces a new description of his or her existing representations. This recoding of information enables the child to think more flexibly and, in drawing behavior, allows the relaxation of a sequential constraint, leading to the eventual depiction of unusual views (Karmiloff-Smith, 1992).

Subsequent drawing research has suggested that pictorial flexibility may be influenced not only by the children's endogenous redescription of mental representation, but also by three possible sources: children's perceptual input (e.g., pictorial models, art, and media), drawing experiences (e.g., availability of drawing material), and learning environments (e.g., instructions, art education, and caregiver-child interactions; Burkitt et al., 2010).

In fact, the possibilities of children to intentionally deviate from a conventional representation of buildings to create an original one in an ecological context are under-investigated (Sameroff, 2010). Indeed, psychological research is mostly conducted in Western cultures on typically developing children in situations of well-being and protection.

The contrastive procedure has inspired test material in a clinical setting (Giordano, 2017) to detect the presence of psychological trauma, but to the best of our knowledge, this pictorial task has not been used to access at-risk children's ideas about their living environment, with few drawing-centered educational experiences, and few opportunities to practice drawing.

Aims of the study. The pictorial flexibility was prompted here by the use of a novel, contractive assignment, and the pictorial theme was a specific relevant element of the human landscape, that is, buildings. The choice of this specific theme-the house in which the children live or would like to live-adds a layer of uniqueness to our data collection, given the particular salience of buildings in the representation of one's daily life. The analysis of the pictorial flexibility was carried out through the evaluation of the variations in the two representations, and of the different graphic solutions through which an effective visual communication was achieved.

The purpose of this study is to investigate whether children between the ages of 8 and 12, who grew up in difficult socioeconomic and cultural circumstances, (1) possess sufficient pictorial flexibility to make drawings of their home, varying the pictorial characteristics of the buildings depicted, in a communicatively effective manner; and (2) the number and type of pictorial strategies with which they pursue the goal of effectively differentiating the buildings depicted, according to the age of the drawers.

We expect that the contrastive task will be effective in stimulating the pictorial flexibility needed to differentiate the picture of their real environment from the picture of the house they would like to live in. We expect this differentiation will be realized to a different extent and in different ways depending on the age of the participants. In particular, we expect that the representational flexibility of the participants will be reflected in the use of fewer pictorial strategies among the younger than among the older ones; we also expect that only the older ones will use more complex differentiation strategies, while the younger ones will use simpler pictorial strategies.

\section{Methods}

Participants. We carried out a study with 200 8-to-12-year-old children, living in a favela in Rio de Janeiro, Brazil. The favela, or shantytown, came into being when squatters occupied vacant land in the city and constructed shanties of salvaged or stolen materials. The lack of infrastructure gives rise to improvised and jury-rigged plumbing and electrical wiring. The favela is also permeated by delinquent activities, first and foremost dominated by illegal drug trafficking. As a result of crowding, unsanitary conditions, poor nutrition, and pollution, disease is rampant in the poorer favelas, and infant mortality rates are high. Although mandatory, school attendance is irregular, and school materials are very limited. The participants were children and adolescents from the favela who attended, even occasionally, the activities of an out-of-school educational center set up in the favela by a team of workers with socio-educational training, free of charge. All participants were asked for their consent to make these drawings for the study, explaining the intent of the activity in language accessible and appropriate to different ages.

The presence of researchers and their activities has been approved by the parents and authorities in the favela. The study was approved by the Department of Education, Languages, Intercultures, Literature and Psychology, University of Florence.

Procedure. The proposed pictorial activity was part of a program to support the development of the favela children's communicative and expressive skills. This program was proposed by the co-author, who worked as a volunteer for social activities at the favela's education center.

Each child was asked to draw two different drawings of a house: one to show what the house they live in looks like, and one to show a house they would like to live in. To draw, the experimenter gave the children white paper $(21 \times 29.7 \mathrm{~cm})$ and a wide variety of drawing materials. The children were asked to draw the buildings as they came to mind, and it was made clear to them that they would not be judged on the beauty of the drawing. The task thus formulated aimed to make the subjects aware of the importance of their thoughts and desires. The order in which the drawing tasks were proposed alternated.

The collection of drawings took place in an ecological context, an ecology that would have been altered or reduced by the administration of formalized tests or other measures of pictorial ability.

Data coding. The 400 drawings collected (two for each child) were classified by two independent judges. First, they assessed the differentiation between the two drawings (the real house vs. the ideal house), deciding which was the real house and which was the ideal house, and indicating the use of which pictorial strategies had been used to indicate the differentiation.

The six strategies used by the children to implement pictorial variation were as follows:

1. Variations in the number of parts: this was assigned when one of the drawings presents a larger number of parts (concerning the whole drawing: house and background) than the other. The coding involved counting the parts of the house on one list (e.g., walls, roofs, windows, doors, balconies, chimneys, etc.) and assigning the number of parts present in each of the two drawings. The scores were given on a dichotomous scale (0-1) according to the presence or absence of the number of element variations. 
2. Variations in the number of colors: as with the previous index, this was assigned when one of the drawings presented a larger number of colors (concerning the whole drawing: house and background) than the other. The coding involved counting the number of colors used. The scores were given on a dichotomous scale (0-1) according to the presence or absence of variation in the number of colors.

3. Variations in size (height and width): assigned when one of the two houses is taller or larger than the other. The coding consisted of measuring centimeters in the height and/or width of each house. The scores were given on a dichotomous scale (0-1), according to the presence or absence of the variation in size.

4. Variations in the number of decorative elements: assigned when there is enrichment with decorative elements applied to the parts of the house, both to the structure and to its environment. The coding involved counting the number of decorative elements used (e.g., the drapes in the windows, the garden, etc.). The scores were given on a dichotomous scale $(0-1)$ according to the presence or absence of the pictorial strategy.

5. Variations in the architectural structure represented: assigned when the two drawings differ in changes in the architectural structure and the functions that the buildings perform. The coding gave the drawn building a scale of increasing architectural value, ranging from shack to palace. A score of 0 was assigned if the buildings belonged to the same category and a score of 1 if there was a difference in architectural value.

6. Variations in the pictorial techniques used: assigned when different technical solutions (pencil, marker, shadows, and transparency) are used for the realization of one of the two drawings. For coding, the drawing is assigned on a scale ranging from the simplest (e.g., pencil) to the most complex (e.g., use of shading). A score of 0 was given if the techniques used in the two drawings were the same, a score of 1 if the techniques were different.

The agreement between the judges was $97 \%$ for the different indices considered. Conflicts were resolved by a third independent judge.

Data analysis and results. Out of the 400 designs reviewed by the judges, $86 \%$ of the drawings were actually two different representations of the two types of houses that could be clearly distinguished (congruent representation). Only $4 \%$ of the drawings were non-congruent representations, and $10 \%$ of drawings did not show any kind of differentiation between the two. According to these results, only 172 pairs of congruent drawings were used to check how many pictorial strategies were used by the children. Of the eliminated drawings, $76 \%$ were drawings of younger children.

First, we controlled for order effects by having each condition occur with the same frequency at each ordinal position. Using ANOVA, we have verified if there were differences between the two condition of order (real vs ideal house and ideal vs real house) and the number of pictorial strategies produced. The order of the task presentation was found to be irrelevant $\left(F_{(1,170)}=0.09, p=\right.$ n.s. $)$.

To test our hypotheses, participants were divided into three groups according to age: $8-9$ years old $(n=50), 10-11$ years old $(n=60)$, and $12-13$ years old $(n=62)$. By age group, it was estimated how many pictorial strategies are present in the drawings that have achieved differentiation effectiveness. Based on each drawing couple, the pictorial strategies were counted and

\begin{tabular}{|c|c|c|c|}
\hline & $8-9 y / 0$ & $10-11 \mathrm{y} / \mathrm{o}$ & $12-13 \mathrm{y} / \mathrm{o}$ \\
\hline Up to two strategies & $92 \%$ & $17 \%$ & $3 \%$ \\
\hline Up to three strategies & $6 \%$ & $78 \%$ & $19 \%$ \\
\hline Four or more strategies & $2 \%$ & $5 \%$ & $78 \%$ \\
\hline
\end{tabular}

\begin{tabular}{|llll|}
\hline $\begin{array}{l}\text { Table } 2 \text { Frequencies of pictorial strategies used in each } \\
\text { drawing by age groups. }\end{array}$ & & & \\
& $\mathbf{8 - 9}$ y/o & $\mathbf{1 0 - 1 1}$ y/o & $\mathbf{1 2 - 1 3}$ y/o \\
\hline Variations in elements & 50 & 60 & 62 \\
Variations in colors & 50 & 60 & 62 \\
Variations in size house & 13 & 41 & 58 \\
Variations in decorative elements & 5 & 18 & 42 \\
Variations in architectural structure & 1 & 12 & 35 \\
Variations in technique & 1 & 4 & 30 \\
Total & 120 & 195 & 275 \\
\hline
\end{tabular}

three levels were identified: (1) drawings that used up to two strategies, (2) drawings that used up to three strategies, and (3) those that used four or more strategies.

Frequency and chi-square analyses were performed to assess the number of differentiating pictorial strategies used by each age group, and whether the association between the number of pictorial strategies used and the age groups was significant. The results showed that the number of pictorial strategies used varied with age $\left(\chi_{(2)}^{2}=191.19, p<0.001\right)$. Specifically, most young children used only up to two pictorial strategies, 10-11year-olds used up to three pictorial strategies, and only older children used four or more pictorial strategies. Table 1 shows the percentages of how many pictorial strategies used in each age group.

To check how often each strategy was used, all strategies were counted within each age group. Specifically, it was found that 8-9-year-olds used a mean of 2 strategies for each pair of drawings; 10-11-year-olds used a mean of 4 strategies; and 12-13-year-olds used a mean of 5 strategies for each pair of drawings. Table 2 shows the frequencies of how often a particular strategy was used in each age group.

A chi-square test was used to verify the association between group age and the typology of pictorial strategy. The results showed a significant association $\left(\chi_{(10)}^{2}=74.38, p<0.001\right)$ (Table 2). Specifically, the results found that younger children use more basic strategies to differentiate between the two drawings, defined as variations in the number of elements, the number of colors, and size. Older children use most sophisticated strategies in a statistically differentiated way, such as variations in the number of decorative elements, in architectural structure, and in the techniques used.

Below are three examples of drawings of a real versus ideal house with the relative pictorial strategies used (Figs. 1, 2, and 3)

\section{Discussion}

In this exploratory study, we investigated whether children's pictorial flexibility, and the ability to perform pictorial representations that deviate from canonical, conventional representations of buildings, were present in children living in favelas. These children live in urban contexts of extreme poverty and with social 


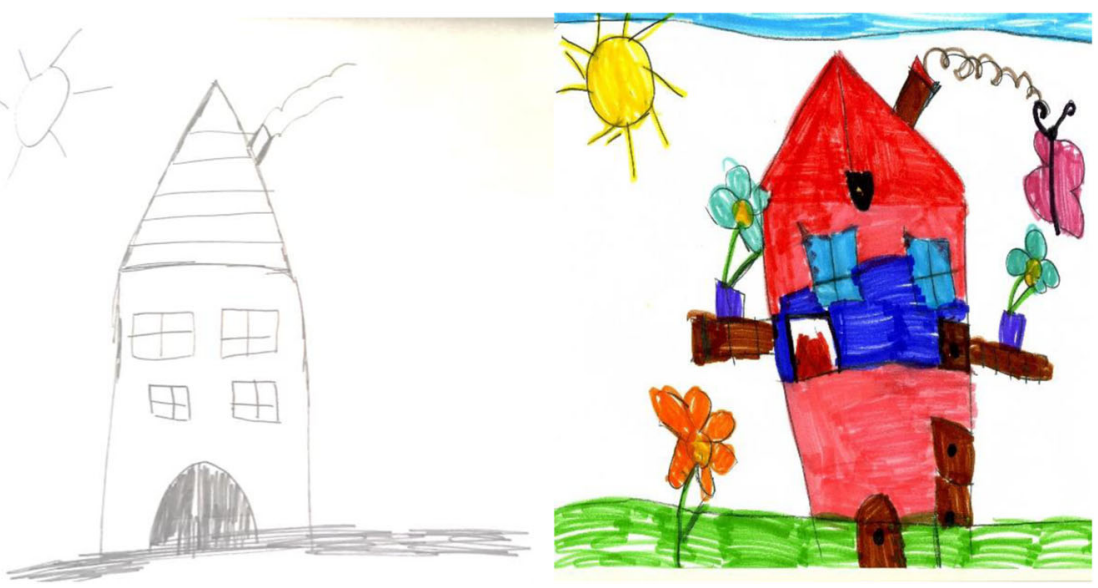

Fig. 1 Drawing of the real house vs. the desired house. F., 8 years old. Example of use of Strategies 1, 2, and 3, and increases in architectural details, colors, and dimensions.
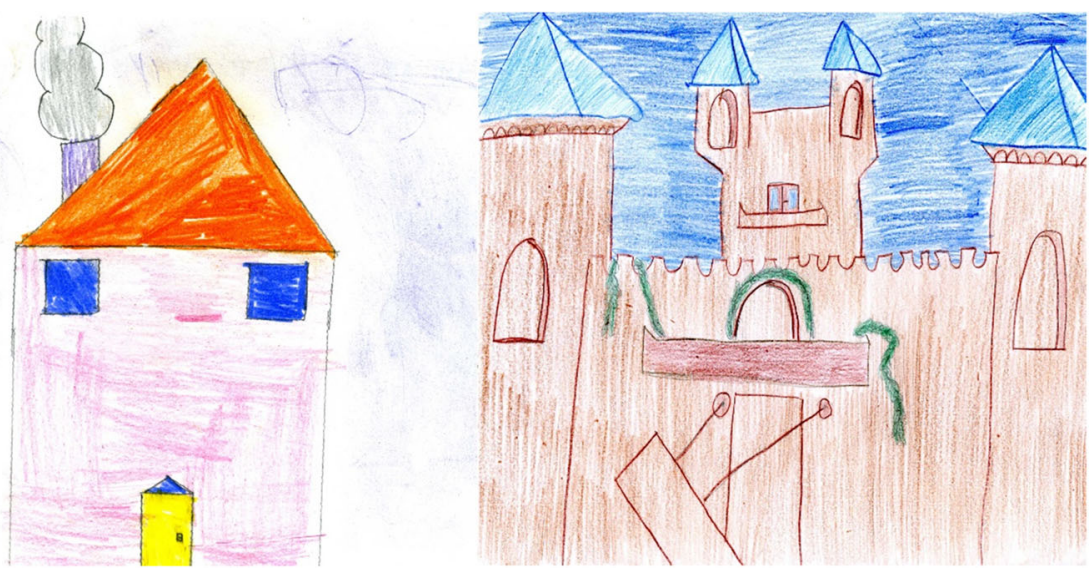

Fig. 2 Drawing of the real house vs. the desired house. F., 10 years old. Example of the use of Strategies 1, 2, 3, and 4, and increases in architectural details, dimensions; change of type of building.

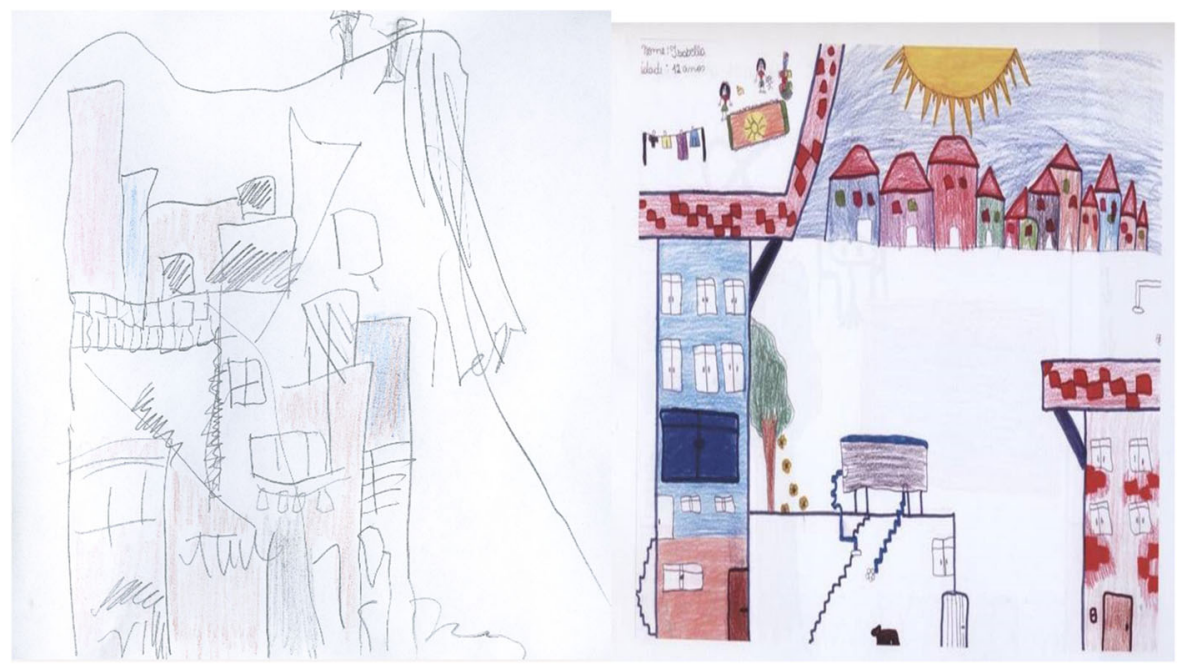

Fig. 3 Drawing of the real house vs. the desired house. F., 12 years old. Example of use of Strategies 1, 2, 4, and 5; increases in architectural details, colors; changes in type of building and pictorial technique.

and cultural disadvantages, who have limited access to schooling and drawing experiences. We also aimed to investigate the developmental pattern that pictorial flexibility would assume in relation to the age of the participants.
In this study, performed with children living in a disadvantaged socioeconomic context, children were asked to draw both the real house and the house in which they would like to live, if their wishes could come true. This request was meant to 
stimulate the pictorial flexibility necessary to mentally transform the reality in which they were immersed and to act on it in an original way. The contrastive drawing task proved to be a powerful way to encourage children to represent and articulate their own ideas and to communicate their ideas to others.

The request to create the two drawings was welcomed by almost all the children, who approached the assignment with commitment and joy. The high degree of confidence with which the judges were then able to distinguish which type of house the young drawer intended to represent each time, confirms that drawing is a particularly suitable communication channel to bring out aspects of children's ideas, which are not easily captured by the most commonly used verbal tools.

The task was accessible to almost all drawers, with the exception of a small number (about 30) of the younger ones, aged 8 and 9 years old. Since at this age the cognitive ability to distinguish the level of reality from that of fantasy is considered mastered, we think that the difficulty in executing two different drawings is of an executive type. In fact, the youngest was still grappling with the execution of a typical, conventional building, a simple and essential house, which they substantially rendered the same way in the second drawing, whatever the theme required. It is possible that this difficulty in performance is exacerbated by the fact that these children live in environments with poor iconography. These children suffer from a poverty of environmental visual models and have little access to iconographic material that they could use as a guide in practicing the possibility of variation (e.g., books designed for children). These results suggest that a lack of drawing practice slows the progression toward drawing mastery, as has been found in research conducted on the execution of other pictorial themes, such as the human figure, by children with little schooling (Martlew and Connolly, 1996).

The results also illustrate that children's age has an influence not only on when representational flexibility emerges, but also on the quantity and quality of the pictorial strategies adopted to clarify the differences between the two types of houses. Younger children use fewer drawing variations than older children, and the ones they perform are the most basic strategies (e.g., variations in the number of parts and colors). It is likely that beginners are strongly pressured by the traditional representation of houses, and for them to deviate from it is very costly from a cognitive and executive point of view.

The difficulty of the task is probably accentuated by the lack of artistic practice these children can have, and by the scarcity of iconographic models to which they are exposed. We know, in fact, that having accessible examples in the environment could instead lighten the ideational load of change, offering ready-made pictorial equivalents, on which to lean when passing from the project to the executive process.

The ability to differentiate between the two types of houses appears to be performed through the targeted use of certain strategies rather than others, according to their ages. The strategies that were mostly used by the youngest subjects to differentiate the two drawings varied in the number of parts, the number of colors, and the size of the elements drawn. These are the simplest pictorial strategies that do not require high planning or advanced executive skills. These simple pictorial devices to transform houses are also accessible to drawers with limited artistic skills. The size of the parts drawn, their dimensions, and the quantity of colors used can be varied during the execution of the drawing, in a summative way, and do not require advanced planning.

Older children, on the other hand, express pictorial flexibility with more complex differentiation strategies, as a change in the pictorial techniques used and a change to the representation of the buildings drawn. These strategies need more elaboration from a cognitive point of view, as they require advanced planning and richer executive skills. These more mature strategies do not limit the differentiation to the perceptual aspects of reality, but portray buildings with more complex qualities, which integrate the perceptual features (shape, number, color of the constituent parts) with the functional and esthetic aspects of buildings.

For the entire sample examined, most of the changes the drawers made benefit the ideal home. The children adhered to the more canonical and essential representations of buildings in drawing their real house, while using their pictorial possibilities to the fullest to show the desirable house. The increase in the number and quality of pictorial details added to complete the ideal structure, and the graphic care with which they were realized. were consistent with the advantages of the desired houses compared to the rudimentary houses they then inhabited. Moreover, the architectural elements that constitute the former were realized in a visually accurate way, thanks to both chromatism and the use of decorative forms, adding to the buildings an esthetic value that goes beyond the strict requirements of functionality.

On the whole, the contrasting pictorial request adopted here was interpreted by the children as an opportunity to give space to their imagination, to distance themselves from their present reality, and create a better place to live, at least on paper. The task prompts children to represent their living environments as scenarios that can change in different time frames, and can facilitate their ability to ask which parts of their current story are permanent and which can possibly change, suggesting that the situation can be looked at in a different way that they have not been considering. In their drawings, they juxtaposed real worlds with possible ones, freeing themselves with their power of imagination from the constraints of everyday life.

These findings tell us something new about the ability of disadvantaged children to draw flexibly and provide some additional understanding of the contribution that drawing can provide to children's resources in coping with adversity. For the children involved, drawing proved to be helpful in expressing feelings that could otherwise be unspeakable and inexpressible. Recreating places on paper reduces the child's helplessness and creates feelings of empowerment. Drawing is a powerful expressive tool that can strengthen children's ability to cope with adversity and increase their cognitive, affective, and communicative resources. For this to happen, however, it is necessary for drawers to have sufficient pictorial skills to effectively represent what they intend to paint and flexibly vary the pictorial theme, departing from its schematic, canonical execution to make room for their own personal ideas about the characteristics of the object drawn.

These results clearly highlight the wishes and hopes that the visual method allows one to express. Inducing people to reflect "visually" can contribute to building a sense of belonging to the place, grasp its specific functions, and color it with functional, cultural, and emotional meanings. The children seem to think, in a resilient way, that beauty and art can contribute to the improvement of their living conditions. Images, more than words, can tell us in which direction the child's gaze is going, and which desirable scenarios reveal themselves in the child's eye.

In this paper, we take a step further to extend drawing into the field of social semiotics (Hodge and Kress, 1997) and into the notion of meaning-making through signs and symbols. This study provides a qualitative repertoire of desires and creative imagination influencing children's experience of their environment, and the results can inspire projects and choices aimed at improving and enriching the domestic and urban scene, attuning it to the participants' expectations. The drawing activity described 
in this paper could be embedded in programs to increase public awareness and strengthen resilience among youth, e.g. to increase children's ability to use drawings to communicate their needs and desires.

\section{Data availability}

The datasets analyzed during the current study are not publicly available but are available from the corresponding author on reasonable request.

Received: 15 January 2021; Accepted: 20 July 2021;

Published online: 09 August 2021

\section{References}

Aysan Y, Oliver P (2001) Housing and culture after earthquakes: a guide for future policy making on housing in seismic areas. Oxford Polytechnic Press, Oxford

Barrett MD, Light PH (1976) Symbolism and intellectual realism in children's drawings. Br J Educ Psychol 46:198-202

Bombi AS, Cannoni E (2003) Saper cambiare. Lo sviluppo di strategie di cambiamento rappresentazionale da 6 a 14 anni. Età Evolutiva 76:72-80

Burkitt E, Jolley R, Rose S (2010) The attitudes and practices that shape children's drawing experience at home and at school. Int J Art Des Educ 29:257-70

Cameron CA, Pinto G, Tapanya S (2014) Scaffolding one Thai youth's drawing toward resilience. Cult Psychol 20(4):453-476. https://doi.org/10.1177/ $1354067 X 14551293$

Chapin LA (2015) Mexican-American boys' positive outcomes and resilience: importance of social support and individual attributes. J Child Fam Stud 24:1791-1799. https://doi.org/10.1007/s10826-014-9982-8

Cherney ID, Seiwert CS, Dickey TM, Flichtbeil JD (2007) Children's drawings: a mirror to their minds. Educ Psychol 26(1):127-142. https://doi.org/10.1080/ 01443410500344167

Clark A (2005) Listening to and involving young children: a review of research and practice. Early Child Dev Care 175(6):489-505

Eisner E (1982) The contribution of painting to children's cognitive development. J Educ 164(3):227-237

Fargas-Malet M, Dillenburger K (2014) Children drawing their own conclusions: children's perceptions of a "postconflict" society. Peace and Confl 20(2):135-149

Fried M (2000) Continuities and discontinuities of place. J Environ Psychol 20:193-205

Giordano F (2017) A qualitative tool for detecting and approaching psychological trauma in children victims of the 2009 Italian earthquake. In Maurer M. (ed.), Child and adolescent mental health (pp. 42-57). IntechOpen. https://doi.org/ $10.5772 / 67364$

Hodge R, Kress G (1997) Social semiotics, style and ideology. In: Coupland N, Jaworski A (eds) Sociolinguistics. Modern linguistics series. Palgrave, London

Huss E, Alhaiga-Taz S (2013) Bedouin children's experience of growing up in illegal villages, versus in townships in Israel: Implications of social context for understanding stress, and resilience in children's drawings. Int J Art Ther 18 (1):10-19. https://doi.org/10.1080/17454832.2012.747217

Izadkhah YO, Gibbs L (2015) A study of preschoolers' perceptions of earthquakes through drawing. In J Disaster Risk Reduct 14:132-139

Karmiloff-Smith A (1990) Constraints on representational change: evidence from children's drawings. Cognition 34:57-83

Karmiloff-Smith A (1992) Beyond modularity: a developmental perspective on cognitive science. MIT Press, Cambridge, MA

Kim S (2005, October) Art therapy emerging response. Disaster News Network. Retrieved January 1, 2006, from http://www.disasternews.net/news/news. php?articleid $=2915$

Laghi F, Baiocco R, Cannoni E, Di Norcia A, Baumgartner E, Bombi AS (2013) Friendship in children with internalizing and externalizing problems: a preliminary investigation with the pictorial assessment of interpersonal relationships. Child Youth Serv Rev 35:1095-1100. https://doi.org/10.1016/j. childyouth.2013.05007

Martlew M, Connolly K (1996) Human figure drawings by schooled and unschooled children in Papua New Guinea. Child Dev 67:2743-2762

Masten AS (2014) Global perspectives on resilience in children and youth. Child Dev 85(1):6-20. https://doi.org/10.1111/cdev.12205

Matthews MH (1985) Young children's representations of the environment: a comparison of techniques. Environ Psychol 5:261-278

Mento C, Modicamore D, La Torre D, Silvestri MC, Rizzo A (2019) Family drawing and psychological vulnerability in Children's representations of parental divorce. Cogent Psychol 6(1):1654723. https://doi.org/10.1080/ 23311908.2019.1654723

Nuttman-Shwartz D, Huss E, Altman A (2010) The experience of forced relocation as expressed in children's drawings. Clin Soc Work J 38(4):397-407

Orr PP (2007) Art therapy with children after a disaster: A content analysis. Arts Psychother 34(4):350-361. https://doi.org/10.1016/j.aip.2007.07.002

Pinto G, Accorti Gamannossi B, Cameron CA (2011) From scribbles to meanings: social interaction in different cultures and the emergence of young children's early drawing. Early Child Dev Care 181(4):425-444. https://doi.org/10.1080/ 03004430903442001

Pinto G, Bombi AS (2007) Children's drawing of friendship and family relationships in different cultures. In: Trautner HM, Milbrath C (eds.) Children's understanding and production of pictures, drawings and art: Theoretical and empirical approaches. Hogrefe \& Huber, Toronto, Ontario, Canada, pp. 121-155

Proshansky HM, Fabian AK (1987) The Development of Place Identity in the Child. In: Weinstein CS, David TG (eds) Space Child. Springer, Boston, MA https://doi.org/10.1007/978-1-4684-5227-3_2

Ring K (2006) Supporting young children drawing: developing a role. Int J Educ Through Art 2(3):195-209

Rutter M (1985) Resilience in the face of adversity: protective factors and resistance to psychiatric disorder. Br J Psychiatr 147:598-611

Sameroff A (2010) A unified theory of development: a dialectic integration of nature and nurture. Child Dev 81(1):6-22. 0009-3920/2010/8101-0002

Thomas G, Silk A (1990) An introduction to the psychology of children's drawings. Harvester Wheatsheaf, London

Winnicott DW (1987) The spontaneous gesture. Harvard University Press, Cambridge, Mass

\section{Competing interests}

The authors declare no competing interests.

\section{Additional information}

Correspondence and requests for materials should be addressed to O.I.

Reprints and permission information is available at http://www.nature.com/reprints

Publisher's note Springer Nature remains neutral with regard to jurisdictional claims in published maps and institutional affiliations.

(i) Open Access This article is licensed under a Creative Commons Attribution 4.0 International License, which permits use, sharing, adaptation, distribution and reproduction in any medium or format, as long as you give appropriate credit to the original author(s) and the source, provide a link to the Creative Commons license, and indicate if changes were made. The images or other third party material in this article are included in the article's Creative Commons license, unless indicated otherwise in a credit line to the material. If material is not included in the article's Creative Commons license and your intended use is not permitted by statutory regulation or exceeds the permitted use, you will need to obtain permission directly from the copyright holder. To view a copy of this license, visit http://creativecommons.org/ licenses/by/4.0/.

(C) The Author(s) 2021 